\title{
Preventing hypoglycaemia: an elusive quest
}

\section{Alan J Sinclair, Srikanth Bellary}

Although our understanding of the causes and consequences of hypoglycaemia has improved greatly, prevention of hypoglycaemia in patients with diabetes remains a major challenge. ${ }^{1}$ Symptomatic mild and severe hypoglycaemia is not only associated with increased risk of cardiovascular events, all-cause hospitalisation, and all-cause mortality ${ }^{2}$ it is also linked to concerns about driving competence, socialisation issues, self -care capacity, serious falls, physical and cognitive status, and impairments in emotional wellbeing and quality of life. ${ }^{3}$ Hypoglycaemia in any adult with diabetes must not only be viewed as a serious adverse drug event, but also as a failure of system process and clinical review by health-care organisations. Over the past decade, recognition of this problem has led to important changes in diabetes management including an increased awareness of hypoglycaemia among health professionals and more focused education for patients. However, based on data presented by Francesco Zacchardi and colleagues ${ }^{4}$ in The Lancet Diabetes \& Endocrinology, the impact of these changes has been limited. The authors report on the trends in admissions with hypoglycaemia in England over a10yearperiod (2005-14) and its impact on length of stay and mortality. There was an initial increase in admissions for hypoglycaemia between 2005 and 2010 (from 7868 in 2005, to 11756 in 2010; 49\% increase), followed by a modest decline in subsequent years (to 10977 in 2014; 39\% increase from baseline); similar trends have been reported in the USA. ${ }^{5}$ Despite varying regional rates of admission, little preadmission review by general practitioners (which might preclude the need for hospital admission in many cases), and the presence of a moderate degree of comorbidity, patients admitted with hypoglycaemia in 2014 were often discharged within 24 h, experienced less in-hospital mortality and fewer were readmitted in the month after discharge when compared with those admitted in 2005 . Thus, our admission policies might now be working well but the flood of admissions into hospital for hypoglycaemia continues.

Although this is promising news for the UK National Health Service, the observed figures are likely to be an underestimate of the true extent of the problem.

Severity of hypoglycaemia varies considerably and not all people experiencing a hypoglycaemic episode present to emergency departments. The occurrence of hypoglycaemia is also often documented by patient report, which, in the case of mild to moderate episodes, remains highly unreliable.

Another important and worrying observation from the data by Zaccardi and colleagues is the high prevalence of hypoglycaemia in adults aged 60 years or older $(72 \%$ of all admissions throughout the study period). In one European study 6 nearly one in five of all emergency admissions to hospital in patients with type 2 diabetes aged 80 years or older was due to hypoglycaemia. A review of the clinical characteristics of those admitted revealed that half were demented, half were in renal failure, and their average $\mathrm{HbA} 1<$ was lower than $40 \mathrm{mmol} / \mathrm{mol}$, all of which represent risk factors for hypoglycaemia. The atypical presentation of hypoglycaemia in older adults, which includes nonspedfic symptoms such as weakness, dizziness, sleepiness, unsteadiness, or confusion, continues to be a neglected observation among health professional staff and may account for both a more severe episode developing and delayed treatment resulting in admission to hospital ${ }^{7}$. Such neuroglycopaenic symptoms can be mistaken for cognitive decline, and might be aggravated in older patients who often experience subjective unawareness of hypoglycaemia and an 
Each health-care organisation should acknowledge that hypoglycaemia has a high public health burden and approach this concern using a cohesive strategy that incorporates patient and health professional education, rationalisation of treatments, use of therapies associated with lower risk of hypoglycaemia, and increased engagement with primary care providers. This approach has to be combined with the implementation of risk minimisation strategies, particularly in older people, that substantially reduce the risk of hypoglycaemia. These strategies can vary from routine evaluation of insulin self-administration abilities to the provision of a hypoglycaemic management kit in care homes, hospices, and palliative care units. Management strategies that involve carers and paramedical services should be explored to support patients outside the hospital and avoid unnecessary hospital admissions.

What is clear is that a target-based management strategy using $\mathrm{HbA}_{1 \mathrm{c}}$ as the sole indicator of treatment success is no longer ideal and in fact might lead to more harm than good. ${ }^{9}$ Alternatively, liberating $\mathrm{HbA}_{1 \mathrm{c}}$ goals alone might not decrease the risk of hypoglycaemia, especially in older adults. ${ }^{10}$ Apart from structured education for all patients on insulin, glinides, and sulfonylureas, a more pragmatic approach would be to set individualised glycaemic targets based on the patient's functional status, ${ }^{11,12}$ which helps to set realistic goals and avoid overtreatment. Future research using prospective studies (rather than retrospective observational ones) are needed to identify the key factors that precipitate hypoglycaemia by age group, and the factors that led to hospital admission. This must be combined with follow-up data that investigate what factors lead to improved clinical outcomes after the event.

1. International Hypoglycaemia Study Group. Minimizing Hypoglycemia in Diabetes. Diabetes Care 2015; 38: $1583-91$.

2. Hsu PF, Sung SH, Cheng HM et al. Association of clinical symptomatic hypoglycaemia with cardiovascular events and total mortality in type 2 diabetes mellitus. Diabetes Care 2012; 36: 894-900.

3. Sinclair A, Dunning T, Rodriguez-Mafias L. Diabetes in older people: new insights and remaining challenges. Lancet Diabetes Endocrinol 2015; 3: 275-85.

4. Zacchardi F, Davies MJ, Dhalwani NN et al. Trends in hospital admissions for hypoglycaemia in England: a retrospective, observational study. Lancet Diabetes Endocrinol 2016; published online June 9. http://dx.doi.org/10.1016/PII.

5. WangJ, Geiss LS, Williams DE, Gregg EW. Trends in emergency department visit rates for hypoglycemia and hyperglycemic crisis among adults with diabetes, United States, 2006-2011. PLoS One 2015; 10: e0134917.

6. Greco D, Pisciotta M, Gambina F, Maggio F. Severe hypoglycaemia leading to hospital admission in type 2 diabetic patients aged 80 yearsor older. Exp Clin Endocrinol Diabetes 2010; 118: 215-19.

7. Thomson FJ, Masson EA, LeemingJT, BoultonAJ. Lack of knowledge of symptoms of hypoglycaemia by elderly diabetic patients. Age Ageing 1991; 20:404-06.

8. BremerJ P, Jauch -Chara K, Hallschmid M, Schmid S, Schultes B. Hypoglycemia unawareness in older compared with middle-aged patients with type 2 diabetes. Diabetes Care 2009; 32: 1513-17.

9. Zoungas S, Patel A, ChalmersJ, et al. Severe hypoglycemia and risks of vascular events and death. N Eg/ J Med 2010; 363: 1410-18.

10. Munshi MN, Segal AR, Suhl E, et al. Frequent hypoglycemia among elderly patients with poor glycemic control. Arch Intern Med 2011; 171:362-34.

11. Inzucchi SE, Bergenstal RM, BuseJB, etal, for the American Diabetes Association (ADA) and European Association for the Study of Diabetes (EASD). Management of hyperglycemia in type 2 diabetes: a patient-centered approach: position statement of the American Diabetes Association (ADA) and the European Association for the Study of Diabetes (EASD). Diabetes Care 2012; 35: $1364-79$.

12. Morley, JE, Sinclair A. Individualising treatment for older people with diabetes. Lancet 2 013; 382: 378-80. 